\title{
Hypercholesterolaemia, signs of islet microangiopathy and altered angiogenesis precede onset of type 2 diabetes in the Goto-Kakizaki (GK) rat
}

\author{
M-H. Giroix • J-C. Irminger • G. Lacraz • C. Noll • S. Calderari • J. A. Ehses • \\ J. Coulaud • M. Cornut • N. Kassis • F. Schmidlin • J-L. Paul • M. Kergoat • N. Janel • \\ P. A. Halban • F. Homo-Delarche
}

Received: 17 March 2011 / Accepted: 23 May 2011 /Published online: 10 July 2011

(C) Springer-Verlag 2011

\begin{abstract}
Aims/hypothesis The adult non-obese Goto-Kakizaki (GK) rat model of type 2 diabetes, particularly females, carries in addition to hyperglycaemia a genetic predisposition towards dyslipidaemia, including hypercholesterol-
\end{abstract}

M-H. Giroix and J-C. Irminger contributed equally to this study.

Electronic supplementary material The online version of this article (doi:10.1007/s00125-011-2223-4) contains peer-reviewed but unedited supplementary material, which is available to authorised users.

M-H. Giroix $(\bowtie) \cdot$ G. Lacraz $\cdot$ J. Coulaud $\cdot$

F. Homo-Delarche $(\bowtie)$

Laboratoire B2PE (Biologie et Pathologie du Pancréas

Endocrine), Unité Biologie Fonctionnelle et Adaptative

(BFA)-EAC CNRS 4413, Université Paris-Diderot,

Bâtiment Lamarck, Case 7104,

5 rue Marie-Andrée Lagroua Weill-Hallé,

75205 Paris Cedex 13, France

e-mail: marie-helene.giroix@univ-paris-diderot.fr

e-mail: fhomodel@orange.fr

J-C. Irminger $\cdot$ M. Cornut $\cdot$ P. A. Halban

Department of Genetic Medicine and Development, CMU,

University of Geneva,

Geneva, Switzerland

C. Noll $\cdot$ N. Janel

Laboratoire de Dérégulation Génique et Différenciation

dans la Trisomie 21 et l'Hyperhomocystéinémie,

Unité Biologie Fonctionnelle et Adaptative (BFA),

Université Paris-Diderot,

Paris, France

\section{S. Calderari}

Laboratoire de Génétique et Génomique Fonctionnelle

de l'Insulinorésistance dans les Systèmes Modèles,

Centre de Recherche des Cordeliers,

Paris, France aemia. As cholesterol-induced atherosclerosis may be programmed in utero, we looked for signs of perinatal lipid alterations and islet microangiopathy. We hypothesise that such alterations contribute towards defective pancreas/islet vascularisation that might, in turn, lead to

\section{J. A. Ehses}

Department of Surgery, Faculty of Medicine,

University of British Columbia and Child \& Family

Research Institute,

Vancouver, BC, Canada

\section{N. Kassis}

Laboratoire HERGE (Homéostasie Energétique et Régulation

Nerveuse et Endocrine), Unité Biologie Fonctionnelle

et Adaptative (BFA), Université Paris-Diderot,

Paris, France

F. Schmidlin $\cdot$ M. Kergoat

Merck Serono,

Chilly-Mazarin, France

J-L. Paul

Assistance Publique-Hôpitaux de Paris (AH-HP),

Hôpital Européen Georges Pompidou,

Laboratoire de Biochimie,

Paris, France

J-L. Paul

Unités de Formation et de recherche (UFR) de Pharmacie,

Université Paris-Sud,

Châtenay-Malabry, France 
decreased beta cell mass. Accordingly, we also evaluated islet inflammation and endothelial activation in both prediabetic and diabetic animals.

Methods Blood, liver and pancreas were collected from embryonic day (E)21 fetuses, 7-day-old prediabetic neonates and 2.5-month-old diabetic GK rats and Wistar controls for analysis/quantification of: (1) systemic variables, particularly lipids; (2) cholesterol-linked hepatic enzyme mRNA expression and/or activity; (3) pancreas (fetuses) or collagenaseisolated islet (neonates/adults) gene expression using Oligo GEArray microarrays targeted at rat endothelium, cardiovascular disease biomarkers and angiogenesis, and/or RT-PCR; and (4) pancreas endothelial immunochemistry: nestin (fetuses) or von Willebrand factor (neonates).

Results Systemic and hepatic cholesterol anomalies already exist in GK fetuses and neonates. Hyperglycaemic GK fetuses exhibit a similar percentage decrease in total pancreas and islet vascularisation and beta cell mass. Normoglycaemic GK neonates show systemic inflammation, signs of islet premicroangiopathy, disturbed angiogenesis, collapsed vascularisation and altered pancreas development. Concomitantly, GK neonates exhibit elevated defence mechanisms.

Conclusions/interpretation These data suggest an autoinflammatory disease, triggered by in utero programming of cholesterol-induced islet microangiopathy interacting with chronic hyperglycaemia in GK rats. During the perinatal period, GK rats show also a marked deficient islet vascularisation in conjunction with decreased beta cell mass.

Keywords Beta cell mass - Cholesterol · Dyslipidaemia . Fetus · Goto-Kakizaki · Islet microangiopathy - Liver . Programming · Type 2 diabetes

$\begin{array}{ll}\text { Abbreviations } \\ \text { AOD } & \text { Antioxidant defence } \\ \text { APOA1 } & \text { Apolipoprotein A1 ( } \mathrm{PGI}_{2} \text {-stabilising factor) } \\ \text { CCL2 } & \begin{array}{l}\text { CC-chemokine ligand-2 or monocyte } \\ \text { chemoattractant protein-1 MCP-1 }\end{array} \\ \text { CCL3 } & \begin{array}{l}\text { CC-chemokine ligand-3 or macrophage } \\ \text { inflammatory protein-1 } \alpha \text { (MIP-1 } \alpha)\end{array} \\ \text { CVD } & \text { Cardiovascular disease } \\ \text { CXCL1 } & \text { CXC-chemokine ligand-1 or chemokine } \\ & \text { GRO1/KC (murine IL-8 equivalent) } \\ \text { E21 } & \text { Embryonic day } 21 \\ \text { ECM } & \text { Extracellular matrix } \\ \text { GK } & \text { Goto-Kakizaki } \\ \text { GSH } & \text { Glutathione } \\ \text { IL-1Ra } & \text { Interleukin-1 receptor antagonist } \\ \text { LCAT } & \text { Lecithin:cholesterol acyltransferase } \\ \text { MMP } & \text { Matrix metalloproteinase } \\ \text { NFKB } & \text { Nuclear factor-KB } \\ \text { OS } & \text { Oxidative stress }\end{array}$

$\mathrm{PGI}_{2} \quad$ Prostaglandin $\mathrm{I}_{2}$ (or prostacyclin)

PON-1 Paraoxonase-1

PTGIS $\quad \mathrm{PGI}_{2}$ synthase or cytochrome P450 8A1 (CYP8A1)

RANKL Receptor activator of nuclear factor-кB ligand or osteoprotegerin ligand (OPGL)

ROS Reactive oxygen species

VWF von Willebrand factor

ZDF Zucker diabetic fatty

\section{Introduction}

The adult Goto-Kakizaki (GK) rat is a spontaneous model of non-obese type 2 diabetes. In addition to hyperglycaemia, the GK rat shows various lipid anomalies such as high circulating NEFA levels, hypertriacylglycerolaemia and hypercholesterolaemia [1]. While hyperglycaemia and hyperlipidaemia induce endothelial dysfunction and inflammation, hypercholesterolaemia alone specifically triggers atherosclerosis $[2,3]$. Recently, we demonstrated the presence of inflammation in pancreatic islets of human type 2 diabetic patients and various rodent models of the disease [4, 5]. We suggested that endothelial activation might underlie inflammation in type 2 diabetic islets, as it does in the eye (retinopathy), kidney (glomerulopathy) and nerves (neuropathy). To test this hypothesis, we treated GK rats with the soluble IL-1 receptor antagonist (IL-1Ra): 1 month of IL-1Ra treatment from onset of diabetes onwards reduced markers of islet endothelial cell activation/dysfunction, inflammation and fibrosis [5, 6]. In vivo, IL-1Ra normalised islet capillaries with decreased evidence of microangiopathy and reduced hyperglycaemia in GK rats as observed in type 2 diabetic patients [7].

Adult GK females are more hypercholesterolaemic than males [1]. In addition maternal cholesterolaemia increases during gestation and a placental gradient of cholesterol is formed between the mother and the fetus [8, 9]. In humans, even temporary maternal hypercholesterolaemia may trigger fatty streak lesions in fetal arteries and accelerate progression of atherosclerosis in normocholesterolaemic children [2]. Such in utero programming has been shown in experimental models of atherosclerosis [3]. We now hypothesise that GK rats present with hypercholesterolaemia combined with an early islet microangiopathy-like reaction during the perinatal period.

In addition, as GK rats exhibit more than a 50\% decrease in beta cell mass from fetal life onwards [10, 11], we hypothesise that defective pancreatic vascularisation is associated with perinatal beta cell mass deficit. Indeed, vessel development, a major component of pancreatic islet development and function, is particularly active around 
birth [12-14] and altered programming of vascularisation has been shown to affect the development and function of various organs [15-17].

To gain further insight into GK dyslipidaemia, we analysed various systemic variables, including different classes of lipids and lipid-related variables at three different ages: embryonic day 21 (E21, hyperglycaemic fetuses) [10], 7 days (normoglycaemic neonates) and 2.5 months after birth (diabetic and insulin-resistant adults) [18]. We also analysed islet and pancreatic gene expression, using Oligo GEArray membranes targeted at rat endothelium, angiogenesis and cardiovascular disease (CVD) biomarkers. Finally, vascularisation was quantified by immunochemistry for endothelial markers.

\section{Methods}

Animals All animal experiments were conducted on agematched GK and Wistar (control) rats from our local colony (Paris, France), in accordance with accepted standards of animal care established by the French National Centre for Scientific Research. GK rats were bred in our own colony at the University Paris-Diderot, together with Wistar control rats from the GK strain derived after backcrossing of animals selected at the upper limit of normal distribution for glucose tolerance [19]. Characteristics of GK rats have been previously described $[1,4-6,10,11,18-21]$. At the indicated age, rats were weighed, bled at time of decapitation and blood, liver and pancreas collected for analysis. Fetuses were taken from pregnant rats fed ad libitum with a standard diet (diet 133; Usine d'Alimentation Rationnelle, Villemoison-sur-Orge, France) at 21 days of gestation (E21) and processed as already described $[10,11]$ after maternal anaesthesia with pentobarbital sodium ( $35 \mathrm{mg} / \mathrm{kg}$ body weight). At E21, the number of fetuses per litter was similar in Wistar and GK rats, $9.0 \pm 0.9(n=9)$ and $8.2 \pm 0.9(n=8)$, respectively, as were the fetal body weights $3.7 \pm 0.1 \mathrm{~g}(n=55)$ and $3.6 \pm$ $0.1 \mathrm{~g}(n=59)$, respectively. The body weight of male GK rats was significantly decreased vs Wistar at both 7 days $(9.1 \pm$ $0.2 \mathrm{~g}, n=26$ vs $15.9 \pm 0.1 \mathrm{~g}, n=36 ; p<0.001)$ and 2.5 months of age ( $271 \pm 3 \mathrm{~g}, n=22$ vs $402 \pm 12 \mathrm{~g}, n=14 ; p<0.001)$.

Systemic variables Basal morning glycaemia was determined with a glucometer. Serum insulin was assayed by ELISA (rat insulin ELISA, cat 10-1124-01, Mercodia, Uppsala, Sweden). Serum NEFA levels were quantified using an enzymatic colorimetric assay (NEFA C, Wako Chemicals, Neuss, Germany). Triacylglycerol, total cholesterol and HDL-cholesterol serum levels were determined by colorimetric assays (Pentra Triglycerid CP kit, Pentra Cholesterol CP kit and HDL-cholesterol direct kit, respectively; ABX Diagnostics, Montpellier, France). Serum ketone levels were measured by quantification of $\beta$-hydroxybutyrate, using the $\beta$-hydroxybutyrate LiquidColor kit (Stanbio Laboratory, Boern TX, USA). Serum alkaline phosphatase activity was quantified using an enzymatic colorimetric assay (Alp CP kit, ABX Diagnostics). Serum leptin was measured using Luminex technology (Millipore, Zug, Switzerland) [5]. Plasma paraoxonase-1 (PON-1) activity and lecithin:cholesterol acyltransferase (LCAT) activity were determined, as previously described [6, 22].

Liver variables Lcat, Pon1 and Apoa1 (encoding apolipoprotein A-1 [APOA1]) mRNA levels were determined by quantitative RT-PCR on a Light Cycler 480 device (Roche Diagnostics, Mannheim, Germany) and PON-1 activity was determined, as previously described $[6,23]$. Primers are listed in electronic supplementary material (ESM) Table 1.

Pancreatic islet isolation and $m R N A$ analysis Analysis of mRNA was performed on pooled pancreases from E21 fetuses. Islets from 7-day-old rats were isolated from the pancreas by a collagenase digestion procedure originally developed for adult rat pancreas and adapted to neonates (see Methods in ESM). Total islet RNA was extracted according to standard protocols using the RNeasy minikit from Qiagen (RNeasy Kit 74104, Hombrechtikon, Switzerland). Islet RNA recovery was around $30-50 \mu \mathrm{g}$ per ten rats. RNA was amplified (two cycles) and biotin-labelled cRNA probes were synthesised using Bioarray High Yield RNA transcript labelling reagents from Enzo Diagnostics (Microsynth, Balgach, Switzerland). Three Oligo GEArray membranes (SABiosciences, Frederick, MD, USA, 128 genes each) were used for hybridisation and were targeted at: (1) rat endothelium (ORN-015.2: permeability and vessel tone, angiogenesis, endothelial cell activation and cell injury); (2) rat cardiovascular disease biomarkers (ORN-037: cell adhesion, immune response, apoptosis, extracellular matrix [ECM] molecules, blood coagulation, circulation, lipid metabolism and genes associated with heart disease); and (3) rat angiogenesis (ORN-024.2: angiogenic factors and other factors involved in angiogenesis, such as cytokines, chemokines, growth factors, adhesion molecules, protease inhibitors, ECM proteins and transcription factors). The procedure was according to protocols from SuperArray Bioscience Corporation (www.superarray. com/). Differential gene expression was confirmed by quantitative RT-PCR (RT: SuperScript II Reverse Transcriptase 11752-050, Invitrogen Life Sciences, Basel, Switzerland and PCR: qPCR Cyber Green kit: Eurogenetec RT-SN10-05, Seraing, Belgium) and quantification performed after normalisation to housekeeping genes as indicated. Primers are listed in ESM Table 1. Further gene classification was performed using investigators' expertise and the PubMed website (www.ncbi.nlm.nih.gov/gene). 
Pancreas immunohistochemistry for endothelial markers Rat islet endothelial proliferation is very high around birth but declines rapidly during the first week postpartum [12]. Nestin was thus used to stain fetal proliferative endothelial cells [24] and von Willebrand factor (VWF) to stain postnatal mature endothelial cells $[4,6]$. For nestin immunohistochemistry, fetal pancreas was fixed in aqueous Bouin's solution and embedded in paraplast. Pancreas sections $(6 \mu \mathrm{m})$ were exposed to mouse anti-nestin $(1 / 50$, SC-33677, Santa Cruz Biotechnology, Santa Cruz, CA, USA) followed by incubation with biotinylated anti-mouse $\operatorname{IgG}(1 / 200$, Vector Laboratories, Burlingame, CA, USA) and processing with the Vectastain ABC kit PK6100 (Vector Laboratories). VWF immunochemistry was performed on neonatal pancreas cryosections $(7 \mu \mathrm{m})$ with rabbit antihuman VWF (1/100, Dako, Glostrup, Denmark) and swine anti-rabbit secondary antibody (1/100, Dako). Immunostained areas were quantified using an Olympus BX40 microscope as previously described by Lacraz et al. [6].
Statistical analysis Data are presented as means \pm SEM. Statistical analyses used the Student's $t$ test for unpaired data. Significance was defined as $p<0.05$ and statistical tendency as $0.05 \leq p \leq 0.10$.

\section{Results}

Systemic metabolic and hormonal anomalies in GK rats as a function of age While GK rats are well known to be normoglycaemic from birth to around weaning at postnatal day 28 [18], GK fetuses present hyperglycaemia at E21 of age (Fig. 1) [10]. E21 GK fetuses were also hypoinsulinaemic, as were 7-day-old neonates, contrary to hyperinsulinaemic and insulin-resistant adult GK rats. As expected, leptinaemia followed the same pattern as insulinaemia [25]. Circulating NEFA levels were similarly low in E21 Wistar and GK fetuses but abnormally elevated in 7-day-old neonates and

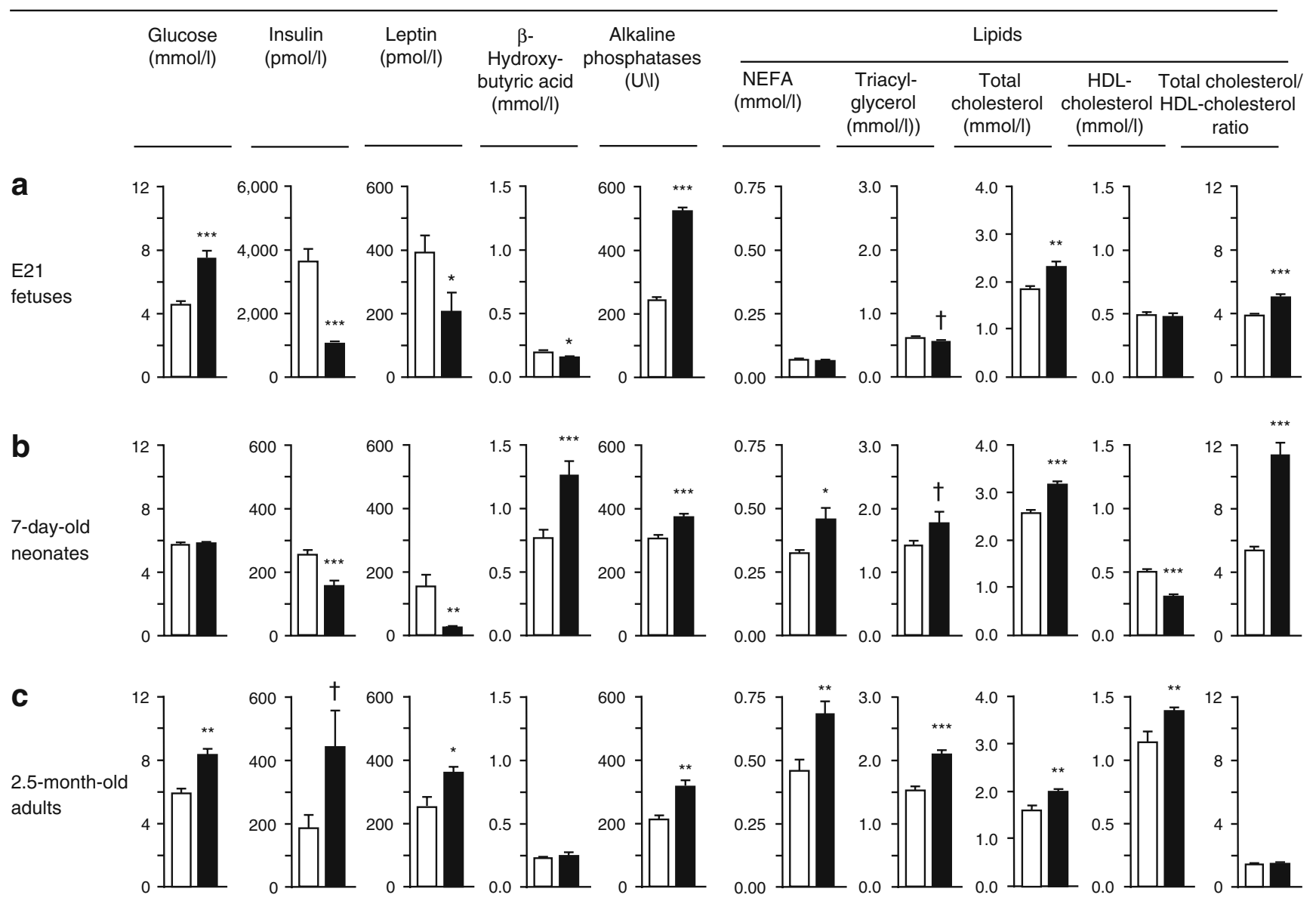

Fig. 1 Systemic metabolic and hormonal variables in Wistar and GK rats as a function of age. All variables were determined in serum of Wistar (white columns) and GK (black columns) rats under fed conditions, except PON-1 activity [23] and fetal insulin measured in plasma as previously described [10]. Values are means \pm SEM for 6-71 fetuses, 10-25 neonates and 4-10 adults in each group of animals. Statistically significant difference $\left({ }^{*} p<0.05, * * p<0.01, * * * p<0.001\right)$ and statistical tendency $\left({ }^{\dagger} 0.05 \leq p \leq 0.10\right)$ vs age-matched Wistar rats, as analysed by Student's $t$ test for unpaired data 
adult GK rats compared with Wistar controls. Systemic triacylglycerol levels followed the same pattern. Circulating levels of the hepatic $\beta$-oxidation marker, $\beta$-hydroxybutyrate, were lower in E21 GK than Wistar fetuses but higher in normoglycaemic 7-day-old GK neonates and identical in adult rats. Importantly, total cholesterol was abnormally elevated in GK rats regardless of age. Although systemic GK and Wistar HDL-cholesterol levels were similar in hyperglycaemic fetuses, HDL-cholesterol levels were decreased in normoglycaemic GK neonates but increased in diabetic GK adults. Finally, alkaline phosphatase levels, reflecting liver and/ or bone anomalies, were increased in GK rats regardless of age. Therefore, dyslipidaemia, in particular systemic cholesterol anomalies, already exists in hyperglycaemic fetuses and normoglycaemic GK neonates.

Systemic and hepatic disturbances of HDL-linked variables in GK rats as a function of age To gain understanding of the very early cholesterol anomalies in GK rats, we measured the evolution of HDL-related variables at different ages (Table 1). Hyperglycaemic E21 GK fetuses exhibited decreased systemic activity of LCAT, the critical enzyme involved in HDL maturation, but normal hepatic Lcat expression. In addition, they also exhibited a drastic increase in hepatic Apoal mRNA compared with Wistar fetuses. ApoAl encodes the predominant HDL protein, but there was no difference in blood- and liver-HDL-associated lipo-lactamase PON-1 (at the level of its activity and/or mRNA level) between GK and Wistar fetuses. By contrast, normoglycaemic 7-day-old GK neonates showed increased systemic LCAT activity with no change in hepatic gene expression. Hepatic ApoA1 expression was downregulated in this group. Circulating and hepatic PON-1 activities were both lowered as compared with age-matched Wistar, with no change in Pon 1 expression. Finally, hyperglycaemic 2.5month-old GK rats were characterised by a normal systemic LCAT activity despite a lowered hepatic Lcat expression. Adult GK rats also displayed elevated circulating PON-1 activity. In liver, this variable was normal despite downregulation of its gene expression. Hepatic ApoAl expression was unchanged in GK adults.

Altogether, these data highlight a pronounced posttranscriptional up/downregulation of hepatic HDL-linked genes and a highly variable pattern of systemic and hepatic HDL-linked variables in GK rats as a function of age and/or metabolic state.

Signs of pancreas or islet inflammation and/or endothelial alterations in GK rats from perinatal life onwards We assessed islet gene expression in Wistar and GK neonates using Oligo GEArray membranes targeted at rat endothelium, CVD and angiogenesis. Over a total of 384 genes, 38 were found to be differentially expressed in 7-day-old prediabetic GK vs control Wistar islets (ESM Table 2). Quantitative RT-PCR confirmed a significant or strong tendency for modulation of 20 genes that we classified into four different groups (Table 2). The first group includes genes involved in inflammation/atherosclerosis that exert mostly deleterious effects with only a few beneficial effects. Most of these genes were upregulated (Casp1, Cc12, the rodent $I l 8$ analogue $\mathrm{Cxcl1}, \mathrm{Il15}, \mathrm{Mmp19}$ ) or tended towards upregulation (Apoa 1, Cpb2, Flt4, Fn1, Il18, Mmp9, Ptgis), while F3, Npy and Tnfrsfl1b were downregulated and $N f \kappa b$ (also known as $N f k b 1$ ) tended to be downregulated.

Table 1 Circulating and hepatic HDL-linked variables in Wistar and GK rats as a function of age

\begin{tabular}{|c|c|c|c|c|c|c|}
\hline \multirow[t]{2}{*}{ Tissue } & \multicolumn{2}{|l|}{ E21 fetuses } & \multicolumn{2}{|l|}{ 7-day-old neonates } & \multicolumn{2}{|c|}{ 2.5-month-old adults } \\
\hline & Wistar & GK & Wistar & GK & Wistar & GK \\
\hline \multicolumn{7}{|l|}{ Blood } \\
\hline PON-1 activity (\%) & $98.4 \pm 4.7(n=5)$ & $98.7 \pm 5.8(n=3)$ & $100.0 \pm 3.8(n=6)$ & $85.5 \pm 1.9^{* *}(n=6)$ & $94.8 \pm 2.8(n=3)$ & $117.9 \pm 7.3^{*}(n=4)$ \\
\hline LCAT activity (\%) & $100.0 \pm 5.6(n=4)$ & $67.2 \pm 3.6^{* *}(n=4)$ & $100.0 \pm 4.5(n=6)$ & $140.3 \pm 9.7^{* *}(n=4)$ & $100.0 \pm 7.4(n=3)$ & $89.0 \pm 2.4(n=3)$ \\
\hline \multicolumn{7}{|l|}{ Liver } \\
\hline Pon1 expression (\%) & $100.0 \pm 64.0(n=3)$ & $98.1 \pm 7.7(n=3)$ & $103.1 \pm 16.9(n=3)$ & $61.3 \pm 13.3(n=5)$ & $100.7 \pm 7.3(n=4)$ & $55.1 \pm 3.8^{* * *}(n=4)$ \\
\hline PON-1 activity (\%) & $100.0 \pm 5.1 \quad(n=3)$ & $93.4 \pm 11.8(n=3)$ & $100.7 \pm 10.7(n=3)$ & $66.8 \pm 0.5^{*}(n=4)$ & $100.0 \pm 4.8(n=7)$ & $94.7 \pm 7.5(n=8)$ \\
\hline Lcat expression (\%) & $100.0 \pm 41.3(n=3)$ & $161.2 \pm 12.0(n=4)$ & $105.5 \pm 21.6(n=4)$ & $198.1 \pm 36.8(n=5)$ & $101.4 \pm 9.4(n=4)$ & $55.9 \pm 9.4^{*}(n=4)$ \\
\hline Apoal expression (\%) & $100.0 \pm 75.0(n=3)$ & $505.4 \pm 96.3^{*}(n=4)$ & $101.1 \pm 10.8(n=3)$ & $62.3 \pm 9.2^{*}(n=5)$ & $101.7 \pm 13.1(n=3)$ & $127.4 \pm 5.8(n=3)$ \\
\hline
\end{tabular}

All variables were determined under fed conditions. Concerning liver gene data, hypoxanthine guanine phosphoribosyl transferase (Hprt) was used as a housekeeping gene (ESM Table 1): no variation was observed between Wistar and GK samples

Values are mean \pm SEM for the number $(n)$ of rats and expressed relative to the mean corresponding value recorded within the same experiments in Wistar rats

${ }^{*} p<0.05,{ }^{*} p<0.01$ vs age-matched Wistar group, as analysed by Student's $t$ test for unpaired data 


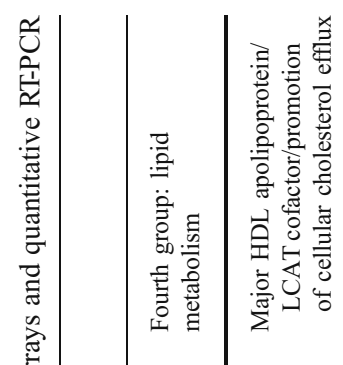

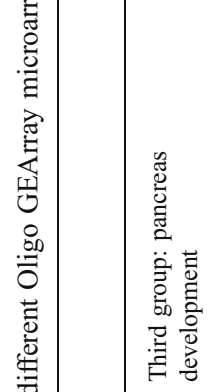

递

:

是

要

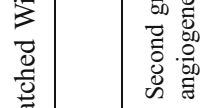

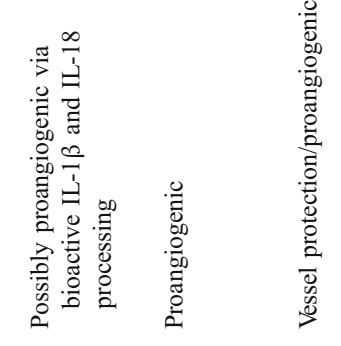

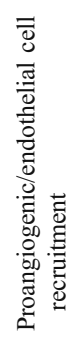

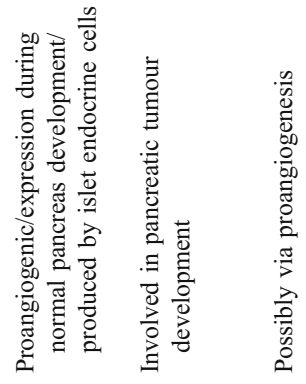

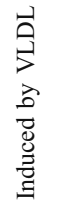

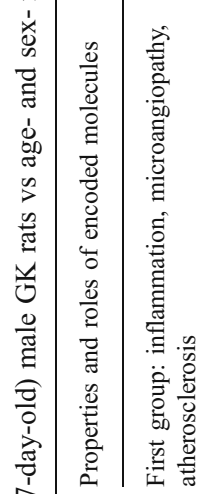

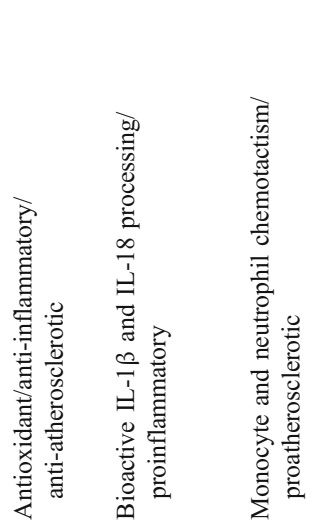
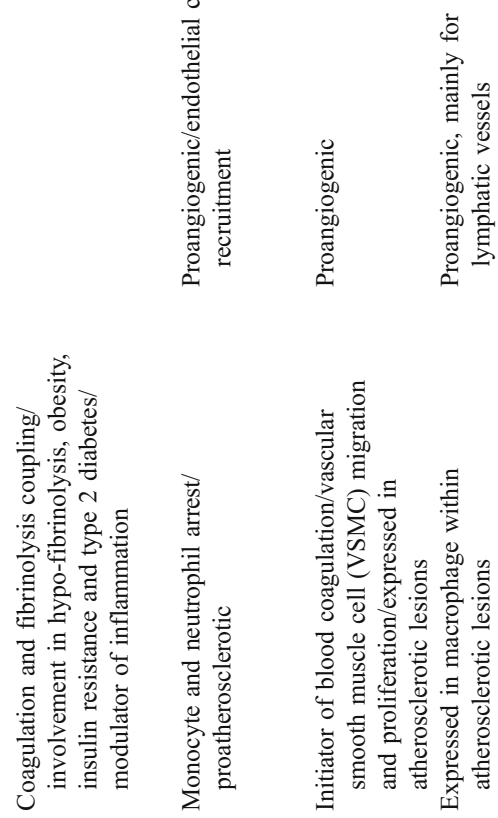

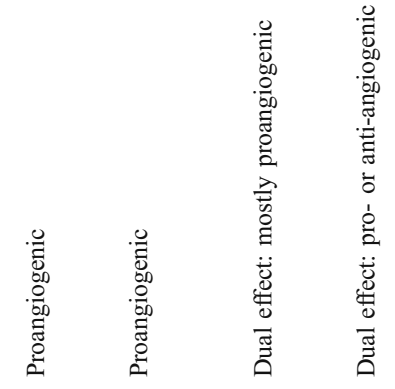

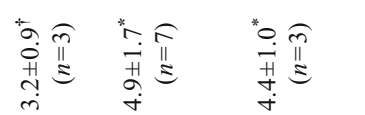
*.
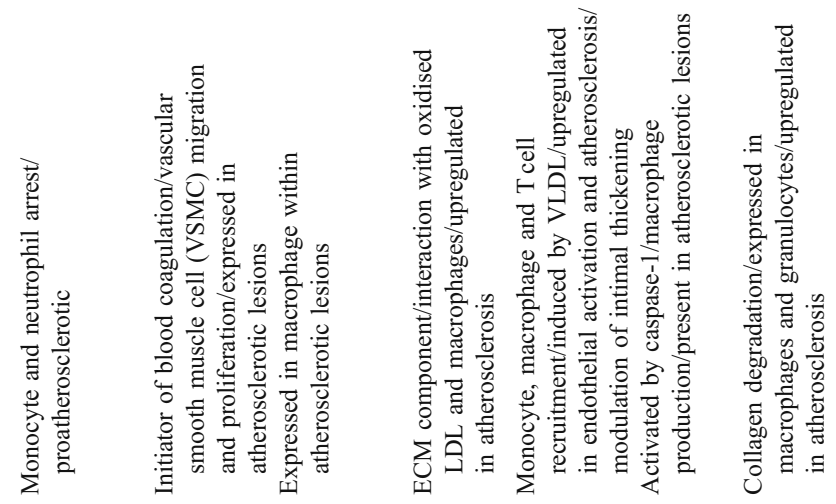

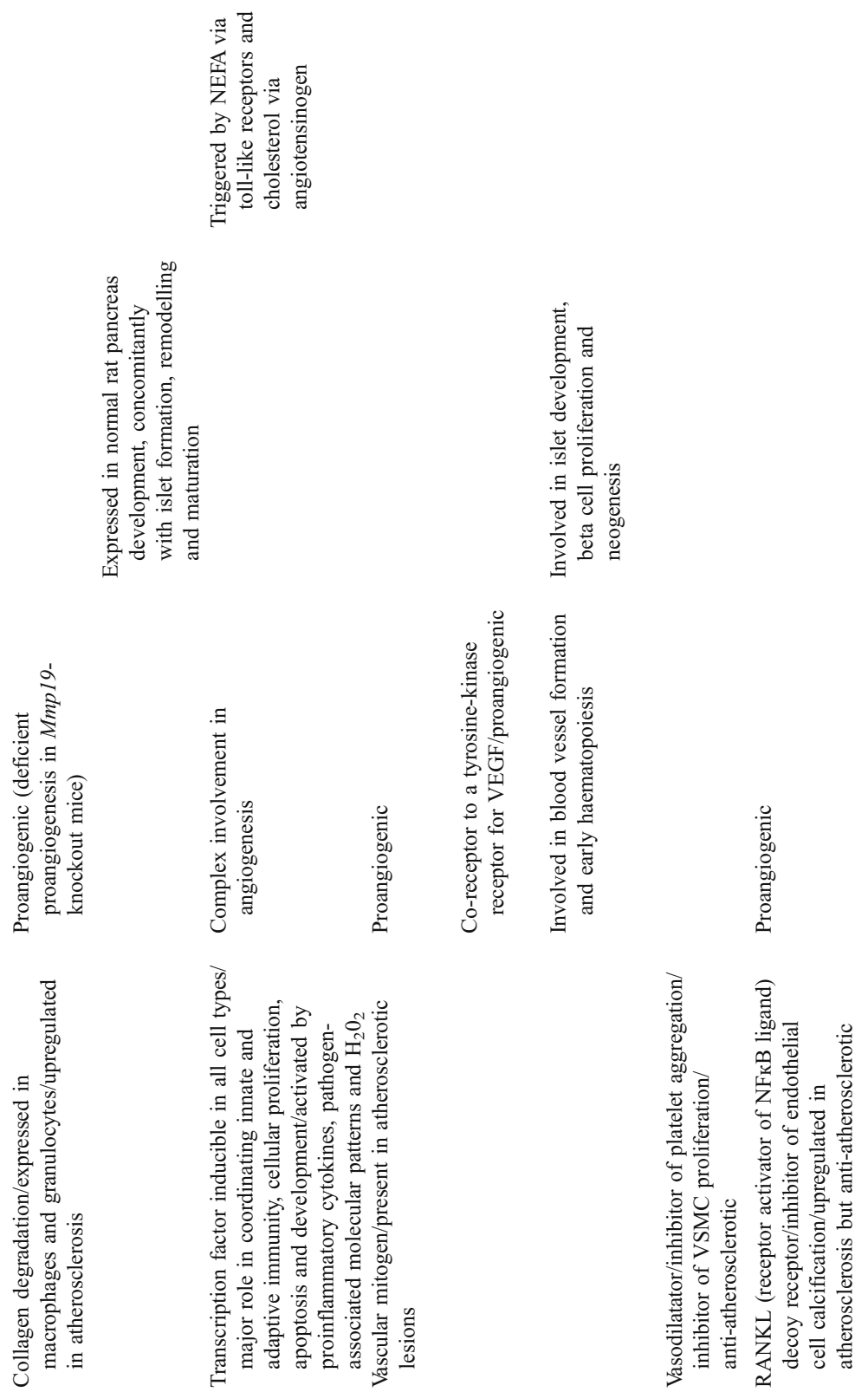

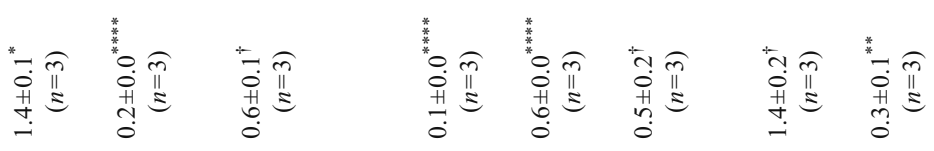

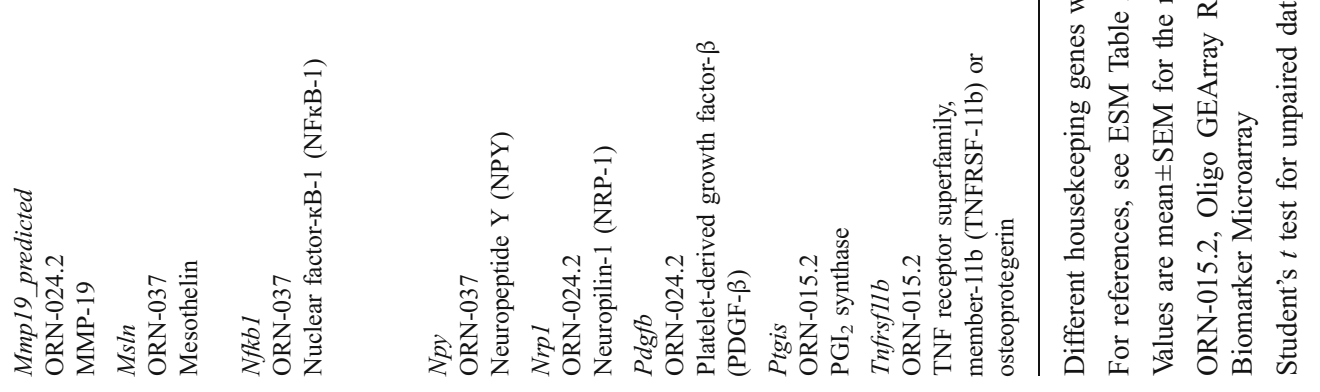


The second group includes angiogenesis-related genes, classified as follows: (1) downregulated proangiogenic genes: Chga, F3, Npy, Nrpl, Pdgf $\beta$ (also known as Pdgfb) and Tnfrsfl1b; and (2) upregulated proinflammatory but also proangiogenic genes: Casp1, Ccl2, Cxcl1, Il15, Ill1, Fn1 and Flt4. The third group includes genes involved in pancreas development: F3, $M \sin$, and $P d g f \beta$ were downregulated, while Flt4 and $F n 1$ tended to be upregulated. The fourth group includes Apoal and Il15, encoding molecules involved in lipid metabolism or being stimulated by lipids.

Next, we investigated whether the expression of endothelium-related genes is already disturbed in the fetal GK pancreas. We selected four inflammatory genes that were upregulated in neonatal GK islets (Casp1, Ill18, Ill5, $\mathrm{Cxcll}$ ) and two downregulated proangiogenic genes ( Nrpl and $N p y$ ) to compare with fetal pancreas gene expression (ESM Fig. 1). All the genes selected, whether up- or downregulated in neonates, were a priori similarly expressed in fetal Wistar and GK pancreases. However, the proangiogenic gene-expression pattern appears to be reversed in fetal GK pancreas and neonatal islets.

As a 'pre-microangiopathy-like state' appears to already characterise islets of normoglycaemic/hypercholesterolaemic GK neonates, we measured the neonatal islet expression of other endothelial activation-related genes which were mostly overexpressed in islets of diabetic GK adults [6]. A comparison of 33 endothelial activation-related genes in islets of normoglycaemic GK neonates and diabetic adults is shown in ESM Fig. 2. More than $50 \%$ of the genes were similarly expressed in neonatal Wistar and GK islets. Six genes were found to be upregulated in neonatal GK islets (Casp1, Ccl2, Cxcl1, Il15, Ill8 and Ptgis) and seven downregulated or with a trend towards downregulation (Cox2, Il6, Hifl $\alpha$ [also known as Hifla], $N f k b$, Nos2, Npy, and Nrp1). By contrast, diabetic GK adults vs Wistar controls showed islet upregulation of almost all endothelial activation-related genes investigated, as well as the expected panel of inflammatory cytokine/ chemokine genes (ESM Fig. 2).

In summary, GK neonates exhibit signs of islet premicroangiopathy, altered angiogenesis and deficient pancreas development, while signs of islet inflammation, endothelial activation and microangiopathy are aggravated by postweaning hyperglycaemia $[5,6]$.

Deficient islet vascularisation in GK fetuses and neonates Altered vascularisation programming might strongly affect organ development and function [15-17] and GK rats present deficient beta cell mass from fetal life onwards $[10,11]$. Rat islet endothelial proliferation is very high around birth but declines rapidly during the first week postpartum [12]. We therefore quantified pancreas and islet vascularisation using nestin and VWF labelling of fetal proliferative and postnatal mature endothelial cells, respectively. Figure $2 \mathrm{a}-\mathrm{e}$ shows that total pancreas section area, total nestin-positive pancreas area, total nestinpositive pancreas area/total pancreas section area and insular nestin-positive area were significantly decreased in GK vs Wistar E21 fetuses (by 29\%, 62\%, 56\% and 55\% respectively). In GK neonates, total VWF-positive pancreas area was $43 \%$ lower than in Wistar controls, total VWF-positive pancreas area/total pancreas section area was unchanged and insular VWF-positive area was drastically decreased (by 90\%) in GK vs Wistar neonates (Fig. 2f-j). Reduction in beta cell mass was also confirmed at both ages (data not shown).

Thus, a marked deficient islet vascularisation is concomitantly observed with decreased beta cell mass in GK rats during the perinatal period.

\section{Discussion}

In this report we show that GK fetuses and neonates exhibit circulating and hepatic lipid anomalies, particularly abnormal cholesterol metabolism. In addition, neonatal GK islets exhibit a pre-microangiopathy-like pattern, associated with signs of altered angiogenesis and disturbed pancreas development. Finally, marked decreased islet vascularisation and beta cell mass are present in GK fetuses and neonates.

Dyslipidaemia from fetal life onwards in GK rats As previously published, hyperglycaemic GK fetuses and normoglycaemic 7-day-old neonates have low basal insulinaemia $[10,11,18]$. GK neonates are normoglycaemic until weaning, because of transiently increased whole body insulin sensitivity [18]. Once GK rats are shifted to a carbohydrate-enriched diet, they exhibit chronic mild hyperglycaemia followed by insulin resistance (hyperinsulinaemia) [18].

A major new finding is that GK rat dyslipidaemia starts in the perinatal period. Increased neonatal NEFA and to a lesser extent triacylglycerol levels result mainly from perinatal GK hypoinsulinaemia. Systemic cholesterol anomalies might result from maternal-fetal GK hyperglycaemia [10, 11]: indeed, a substantial body of data provides a link between glucose and cholesterol metabolism [26, 27]. Moreover, low perinatal GK leptinaemia might also contribute to cholesterol anomalies by lowering the expression/activity of acyl-coenzyme A:cholesterol acyltransferase-1, thus reducing cholesteryl ester accumulation in macrophages and foam cell formation [28]. These perinatal data add to the knowledge on cholesterol metabolism in diabetic GK adults and their gene linkage [1]. 
a
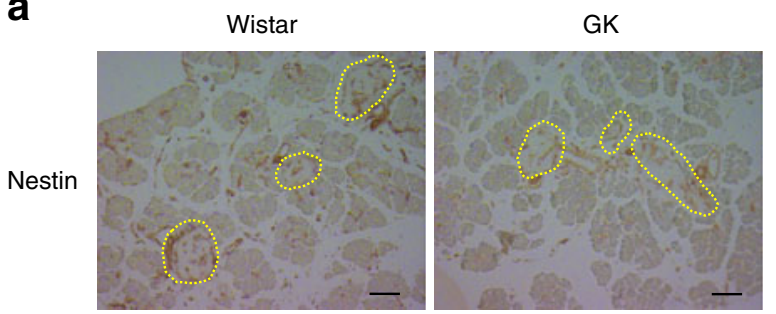

$\mathbf{f}$

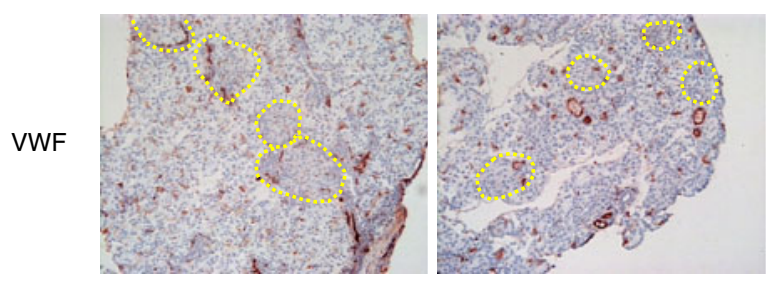

b

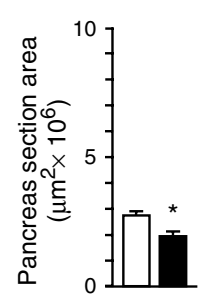

g

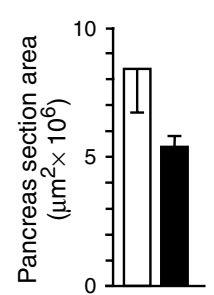

C

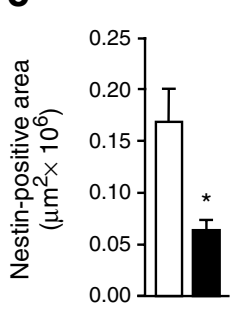

h

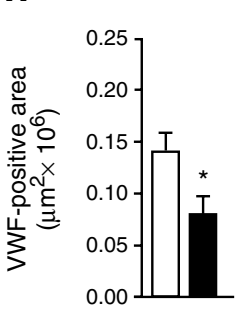

d

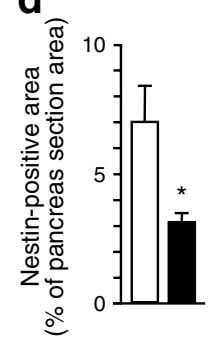

i

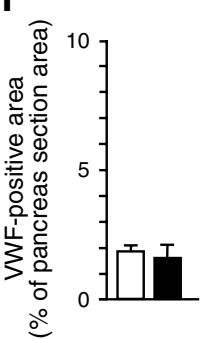

e

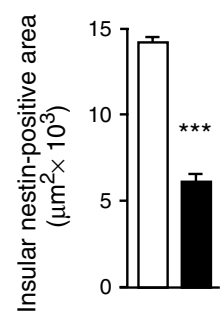

j

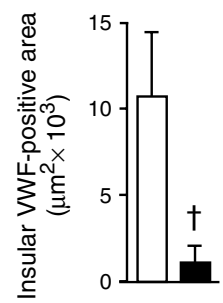

Fig. 2 Pancreas immunochemistry for endothelial markers in Wistar and GK E21 fetuses and 7-day-old neonates. a Nestin immunochemical staining of fetal proliferative endothelial cells [24]; in particular in Wistar islets, note that nestin labelled peri- and intra-islet vascularisation as described elsewhere $[12,24]$ and numerous capillaries are also observed in the mesenchyme or near the acini. Scale bar, $50 \mu \mathrm{m}$. b-e Immunolabelled pancreas or islet area for nestin in Wistar (white columns) and GK (black columns) rats was quantified for each pancreas section and expressed relative to the corresponding pancreas section area or total islet area, respectively ( $n=4$ fetuses): (b) pancreas section area; (c) nestin-positive area; (d) nestin-positive/pancreas section area; (e) insular nestin-positive area. f VWF immunochemical staining

Early disturbances in HDL metabolism in GK rats GK fetuses are hyperglycaemic because of maternal diabetes $[10,11]$. Hyperglycaemia induces deleterious protein glycation, as described for the predominant HDL protein APOA1 or HDL-maturation protein LCAT [29, 30]. Hyperglycaemia also induces reactive oxygen species (ROS) production, which is responsible for oxidative stress (OS), and therefore represents the pathogenic mechanism unifying diabetes and CVD [31]. Thus, in GK fetuses, glycation of LCAT might decrease its systemic activity with attempted compensation through increased hepatic mRNA levels. Similarly, glycation of APOA1 may cause the sharp in vivo increase in liver Apoal expression, while high glucose concentrations in vitro reduce Apoal expression in hepatocytes [27]. The absence of modification of blood and liver HDL-associated lipo-lactamase PON-1 is probably due to the very low PON-1 activity in rodents before birth. Indeed, PON-1 activity develops rapidly from birth to 21 days of age [32].

In 7-day-old normoglycaemic GK rats, the decrease in both systemic and hepatic PON-1 activities might result from prenatal PON-1 glycation, only visible after birth [32, 33]. In addition, inhibition of liver PON-1 activity is an of postnatal mature endothelial cells $[4,6]$. Magnification $\times 250$. $\mathbf{g}-\mathbf{j}$ Immunolabelled pancreas or islet area for VWF in Wistar (white columns) and GK (black columns) rats was quantified for each pancreas section and expressed relative to the corresponding pancreas section area or total islet area, respectively ( $n=5-6$ neonates per group): (g) pancreas section area; (h) VWF-positive area; (i) VWF-positive/pancreas section area; (j) insular VWF-positive area. In pancreas sections stained for endothelial markers, the islet border is defined by a dashed line. Values are means \pm SEM. $* p<0.05$ and $* * * p<0.001$ and statistical tendency $\left({ }^{\dagger} 0.05 \leq p \leq 0.10\right)$ vs age-matched Wistar rats, as analysed by Student's $t$ test for unpaired data

early biochemical change linked to increased lipid peroxidation and liver damage [34]. Notably, oxysterols increase total liver peroxides, IL-8 and CC-chemokine ligand-2 (CCL2) [35]. Free cholesterol decreases hepatic glutathione (GSH) levels, sensitises hepatocytes to TNF- $\alpha$ and leads to inflammation [36]. The findings of maternal/fetal hyperglycaemia and concomitant fetal hypercholesterolaemia lead us to suggest that OS is at work in perinatal GK liver. This hypothesis is reinforced by the existence of systemic low erythrocyte GSH content and elevated CCL2, CCchemokine ligand-3 (CCL3) and CXC-chemokine ligand-1 (CXCL1) levels in GK neonates [20].

Low neonatal GK circulating HDL-cholesterol levels and high total cholesterol/HDL-cholesterol ratio might result from decreased circulating fetal LCAT activity. However, return to normoglycaemia after birth and attempts to increase liver Lcat mRNA levels may improve LCAT activity in GK neonates. The postnatal liver Apoal downregulation that parallels low systemic HDL levels in normoglycaemic GK neonates might reflect a delayed effect of fetal hyperglycaemia [27]. Such perinatal adaptive regulation of GK liver variables highlights an early 'programmed' and deleterious interaction of carbohydrates 
and lipids on hepatic offspring lipid metabolism, as is observed with maladapted maternal diets [37].

In GK adults systemic signs of OS, as assessed by elevated oxidised GSH levels in erythrocytes, develop after diabetes onset [20]. However, high circulating PON-1 activity and $\alpha$-tocopherol concentrations suggest that diabetic GK adults have installed systemic antioxidant defences (AODs). This may consequently return systemic GK chemokine levels to age-matched Wistar levels [6]. Similarly, normalisation of total cholesterol/HDL-cholesterol ratio in diabetic GK adults might result from stimulation of neonatal systemic GK LCAT activity. Indeed, adult GK rats overexpressed some AOD genes in liver (Lacraz, unpublished data) and overall defence involving islet antioxidant/cAMP/ antiapoptotic pathway, which confers beta cell protection by decreasing ROS and apoptosis [20, 21]. ESM Table 3 shows that systemic HDL-cholesterol levels and total cholesterol/ HDL-cholesterol ratio correlated negatively and positively, respectively, with pancreas/islet expression of Casp1, IL18 and IL15. These data are in agreement with the wellrecognised anti-inflammatory effects of HDL [38].

Signs of defective islet angiogenesis, development and vascularisation in GK fetuses and/or neonates A major feature of GK rats from perinatal life onwards (except surprisingly at E21) is a markedly lower body weight than Wistar controls, despite maternal/fetal hyperglycaemia $[10$, 11, 18]. However, low perinatal levels of trophic factors, like insulin, IGFs and leptin, might be responsible for low postnatal GK body weight [11, 39-41]. Low perinatal GK leptinaemia is associated with a low fat/body mass percentage [18]. Furthermore, a defective IGF2 and IGF1 receptor protein production in GK pancreatic rudiment as early as E13.5 has been reported [11]. Insulin, IGFs and leptin also stimulate angiogenesis, a crucial process for adapted development [39-41]. Our neonatal data highlight deficient GK islet expression of genes, such as F3, Msln, Pdgf $\beta$, Chga, Npy and Nrp1, encoding molecules involved in pancreas development and/or angiogenesis. Lammert hypothesised that changes within the vascular system may act very early in triggering type 2 diabetes. This is based on the role of blood vessels in islet development and the vascular-related functions of most genes involved in human disease [14]. Accordingly, we show that beta cell-massdeficient GK fetuses and neonates also exhibit marked islet vascularisation defects, as assessed by nestin and VWF immunostaining, respectively $[10,11,24]$. The concomitant downregulation of Npy and Nrp1 and collapse of vascularisation in neonatal GK islets should be noted. Indeed, systemic GK glucose and alkaline phosphatase levels correlated positively with the pancreas/islet expression of both Nrpl and Npy, while NEFA levels correlated negatively with the expression of both these factors (ESM
Table 3). These data suggest a link between metabolic disturbances and angiogenesis. Adult GK rats are unable to adapt beta cell mass and vascularisation after beta cell depletion [42]. This might partly result from GK rats being unable to upregulate islet Vegf, in contrast to 7-12-week-old Zucker diabetic fatty (ZDF) rats [43]. The expression/ signalling of angiogenic factor is also abnormal in GK myocardial microvasculature [44]. Thus, the non-obese GK rat model resembles human non-obese type 2 diabetic patients, who exhibit a genetic basis for low birthweight and deficient vascularisation $[14,45]$.

'Pre-microangiopathy-like'state in islets of normoglycaemic $G K$ neonates We show here that several genes involved in inflammation and atherosclerosis are overexpressed in neonatal GK islets. Importantly this is concomitant with high islet ROS levels, elevated GSH oxidised state and altered Gpxl, Gsr and Trx [also known as Txn1 or Txn] expression, as previously described [20]. These islet conditions might result from the proinflammatory effects of fetal hyperglycaemia and perinatal hypoinsulinaemia and dyslipidaemia, particularly proatherosclerotic hypercholesterolaemia. However, the neonatal islet inflammation/endothelial activation is markedly lower than in diabetic adult GK islets. Indeed, after weaning, GK islets are boosted by chronic hyperglycaemia and hyperlipidaemia, leading to full blown islet endothelial activation, inflammation, fibrosis and beta cell deterioration [4-6, 20]. The positive correlation between systemic insulin levels and pancreatic/islet Cxcll expression (ESM Table 3) might reflect decreased anti-inflammatory effects of insulin due to emergence of insulin resistance. While fetal GK hypercholesterolaemia might trigger an in utero cholesterolinduced disease programming as in humans [3], taken together these data highlight a complex programming of metabolically induced endothelial and islet alterations. Moreover, it has been recently suggested that atherosclerosis and type 2 diabetes are inflammasome-mediated autoinflammatory diseases $[46,47]$. The overexpression of genes encoding caspase-1, IL-1, IL-18, nuclear factor-кB (NFкB), and many inflammatory proteins in neonatal and/or adult GK rat islet physiopathology reinforces this hypothesis.

Attempts to compensate perinatal deleterious effects in islets of prediabetic GK neonates First, neonatal islets are the site of: upregulation of most genes encoding proinflammatory cytokines and chemokines with proangiogenic properties, possibly reflecting a repair process; and upregulation or trends towards upregulation of Flt4, which participates in the recruitment of VEGF-A-secreting macrophages involved in angiogenesis [48], F3 and Mmp19 [49, 50]. Second, those islets show a trend towards upregulation of genes involved in vascular protection, like those encoding prostaglandin $\mathrm{I}_{2}$ 
$\left(\mathrm{PGI}_{2}\right)$ synthase (PTGIS), which synthesises the vasodilatator agent $\mathrm{PGI}_{2}$, and APOA1, also known as $\mathrm{PGI}_{2}$-stabilising factor [51]. Intriguingly, Apoal expression is assumed to occur in liver and intestine only, but has also been observed in human fetal pancreas [52]. Third, neonatal GK islets exhibit a downregulation of inflammatory NFKB-related genes, such as Cox2, Il6, Nos 2 and $N f k b$ itself [53]. The mechanism of this phenomenon deserves further investigation. At a later stage, as mentioned above, islets of adult diabetic rats partly protect themselves against OS through large adaptive AOD upregulation [20].

In conclusion, dyslipidaemia and elevated systemic/ hepatic cholesterol levels are already present in hyperglycaemic GK fetuses and normoglycaemic GK neonates. This is associated with signs of neonatal islet premicroangiopathy, altered angiogenesis and pancreas development and deficient islet vascularisation. GK rats also present various forms of defence mechanisms potentially able to limit disease progression, while deficient angiogenesis might prevent full beta cell recovery. This pathophysiological sequence may help to understand why low birthweight infants, who exhibit several similar metabolic disorders [54], are at risk of developing insulin resistance, type 2 diabetes and CVD $[3,55]$.

Acknowledgements These studies were funded by the Centre National de la Recherche Scientifique, the Fond National Suisse de la Recherche Scientifique and the EFSD/MSD European Research Programme. The funders had no role in study design, data collection and analysis, decision to publish, or preparation of the manuscript. G. Lacraz and C. Noll were recipients of doctoral fellowships from the Ministère de l'Education Nationale, de l'Enseignement Supérieur et de la Recherche, France. We are grateful to D. Bailbé (Université ParisDiderot, BFA-CNRS 4413 Unit) for her expert assistance in the GK rat breeding (GK/Par colony). Parts of this work were presented at EASD Meetings (2008 and 2009), Keystone Symposium 'Dissecting the Vasculature: Function, Molecular Mechanisms and Malfunction' (2009), 2nd International Brussels Pancreatic Islet Symposium: pancreatic islet research from fundamental to clinical aspects (2009) and the 49th Meeting of The American Society for Cell Biology (2009).

Conception and design, or data analysis and interpretation of data: M-HG, J-CI, GL, CN, SC, JAE, JC, MC, NK, FS, J-LP, MK, NJ, PAH, FH-D; drafting article or revising it critically for important intellectual content: M-HG, J-CI, GL, CN, SC, JAE, JC, MC, NK, FS, J-LP, MK, NJ, PAH, FH-D; final approval of the version to be published: $\mathrm{M}-\mathrm{H} \mathrm{G}$, J-CI, GL, CN, SC, JAE, JC, MC, NK, FS, J-LP, MK, NJ, PAH, FH-D.

Duality of interest The authors declare that there is no duality of interest associated with this manuscript.

\section{References}

1. Argoud K, Wilder SP, McAteer MA et al (2006) Genetic control of plasma lipid levels in a cross derived from normoglycaemic Brown Norway and spontaneously diabetic Goto-Kakizaki rats. Diabetologia 49:2679-2688
2. Napoli C, Glass CK, Witztum JL et al (1999) Influence of maternal hypercholesterolaemia during pregnancy on progression of early atherosclerotic lesions in childhood: Fate of Early Lesions in Children (FELIC) study. Lancet 354:1234-1241

3. Palinski W, Nicolaides E, Liguori A, Napoli C (2009) Influence of maternal dysmetabolic conditions during pregnancy on cardiovascular disease. J Cardiovasc Transl Res 2:277-285

4. Homo-Delarche F, Calderari S, Irminger JC et al (2006) Islet inflammation and fibrosis in a spontaneous model of type 2 diabetes, the GK rat. Diabetes 55:1625-1633

5. Ehses JA, Lacraz G, Giroix MH et al (2009) IL-1 antagonism reduces hyperglycemia and tissue inflammation in the type 2 diabetic GK rat. Proc Natl Acad Sci USA 106:13998-14003

6. Lacraz G, Giroix MH, Kassis N et al (2009) Islet endothelial activation and oxidative stress gene expression is reduced by IL-1Ra treatment in the type 2 diabetic GK rat. PLoS One 4:e6963

7. Larsen CM, Faulenbach M, Vaag A et al (2007) Interleukin-1-receptor antagonist in type 2 diabetes mellitus. N Engl J Med 356:1517-1526

8. Herrera E, Amusquivar E, Lopez-Soldado I, Ortega H (2006) Maternal lipid metabolism and placental lipid transfer. Horm Res 65(Suppl 3):59-64

9. Marseille-Tremblay C, Ethier-Chiasson M, Forest JC et al (2008) Impact of maternal circulating cholesterol and gestational diabetes mellitus on lipid metabolism in human term placenta. Mol Reprod Dev 75:1054-1062

10. Serradas P, Gangnerau MN, Giroix MH, Saulnier C, Portha B (1998) Impaired pancreatic beta cell function in the fetal GK rat. Impact of diabetic inheritance. J Clin Invest 101:899-904

11. Calderari S, Gangnerau MN, Thibault M et al (2007) Defective IGF2 and IGF1R protein production in embryonic pancreas precedes beta cell mass anomaly in the Goto-Kakizaki rat model of type 2 diabetes. Diabetologia 50:1463-1471

12. Johansson M, Andersson A, Carlsson PO, Jansson L (2006) Perinatal development of the pancreatic islet microvasculature in rats. J Anat 208:191-196

13. Nikolova G, Jabs N, Konstantinova I et al (2006) The vascular basement membrane: a niche for insulin gene expression and Beta cell proliferation. Dev Cell 10:397-405

14. Lammert E (2008) The vascular trigger of type II diabetes mellitus. Exp Clin Endocrinol Diabetes 116:S1-S5

15. Cambonie G, Comte B, Yzydorczyk C et al (2007) Antenatal antioxidant prevents adult hypertension, vascular dysfunction, and microvascular rarefaction associated with in utero exposure to a low-protein diet. Am J Physiol Regul Integr Comp Physiol 292: R1236-1245

16. Torrens C, Kelsall CJ, Hopkins LA et al (2009) Atorvastatin restores endothelial function in offspring of protein-restricted rats in a cholesterol-independent manner. Hypertension 53:661-667

17. Watkins AJ, Lucas ES, Torrens C et al (2010) Maternal low-protein diet during mouse pre-implantation development induces vascular dysfunction and altered renin-angiotensin-system homeostasis in the offspring. Br J Nutr 103:1762-1770

18. Movassat J, Bailbe D, Lubrano-Berthelier C et al (2008) Follow-up of GK rats during prediabetes highlights increased insulin action and fat deposition despite low insulin secretion. Am J Physiol Endocrinol Metab 294:E168-175

19. Goto Y, Kakizaki M, Masaki N (1975) Spontaneous diabetes produced by selective breeding of normal Wistar rats. Proc Jpn Acad 51:80-85

20. Lacraz G, Figeac F, Movassat J et al (2009) Diabetic beta-cells can achieve self-protection against oxidative stress through an adaptive up-regulation of their antioxidant defenses. PLoS One 4:e6500

21. Lacraz G, Figeac F, Movassat J, Kassis N, Portha B (2010) Diabetic GK/Par rat beta-cells are spontaneously protected against $\mathrm{H}_{2} \mathrm{O}_{2}$-triggered apoptosis. A cAMP-dependent adaptive response. Am J Physiol Endocrinol Metab 298:E17-27 
22. Jonas A (2000) Lecithin cholesterol acyltransferase. Biochim Biophys Acta 1529:245-256

23. Noll C, Hamelet J, Ducros Vet al (2009) Resveratrol supplementation worsen the dysregulation of genes involved in hepatic lipid homeostasis observed in hyperhomocysteinemic mice. Food Chem Toxicol 47:230-236

24. Suzuki S, Namiki J, Shibata S, Mastuzaki Y, Okano H (2010) The neural stem/progenitor cell marker nestin is expressed in proliferative endothelial cells, but not in mature vasculature. J Histochem Cytochem 58:721-730

25. Forhead AJ, Fowden AL (2009) The hungry fetus? Role of leptin as a nutritional signal before birth. J Physiol 587:1145-1152

26. Albrecht C, Simon-Vermot I, Elliott JI et al (2004) Leukocyte ABCA1 gene expression is associated with fasting glucose concentration in normoglycemic men. Metabolism 53:17-21

27. Dey A, Chandrasekaran K (2009) Hyperglycemia induced changes in liver: in vivo and in vitro studies. Curr Diabetes Rev 5:67-78

28. Hongo S, Watanabe T, Arita S et al (2009) Leptin modulates ACAT1 expression and cholesterol efflux from human macrophages. Am J Physiol Endocrinol Metab 297:E474-482

29. Fournier N, Myara I, Atger V, Moatti N (1995) Reactivity of lecithin-cholesterol acyl transferase (LCAT) towards glycated high-density lipoproteins (HDL). Clin Chim Acta 234:47-61

30. Nobecourt E, Tabet F, Lambert G et al (2010) Nonenzymatic glycation impairs the antiinflammatory properties of apolipoprotein A-I. Arterioscler Thromb Vasc Biol 30:766-772

31. Brownlee M (2001) Biochemistry and molecular cell biology of diabetic complications. Nature 414:813-820

32. Soran H, Younis NN, Charlton-Menys V, Durrington P (2009) Variation in paraoxonase-1 activity and atherosclerosis. Curr Opin Lipidol 20:265-274

33. Ikeda Y, Suehiro T, Arii K, Kumon Y, Hashimoto K (2008) High glucose induces transactivation of the human paraoxonase 1 gene in hepatocytes. Metabolism 57:1725-1732

34. Camps J, Marsillach J, Joven J (2009) Measurement of serum paraoxonase-1 activity in the evaluation of liver function. World $\mathrm{J}$ Gastroenterol 15:1929-1933

35. Ferre N, Martinez-Clemente M, Lopez-Parra M et al (2009) Increased susceptibility to exacerbated liver injury in hypercholesterolemic ApoE-deficient mice: potential involvement of oxysterols. Am J Physiol Gastrointest Liver Physiol 296:G553-562

36. Alkhouri N, Dixon LJ, Feldstein AE (2009) Lipotoxicity in nonalcoholic fatty liver disease: not all lipids are created equal. Expert Rev Gastroenterol Hepatol 3:445-451

37. Qasem RJ, Cherala G, D'Mello AP (2010) Maternal protein restriction during pregnancy and lactation in rats imprints long-term reduction in hepatic lipid content selectively in the male offspring. Nutr Res 30:410-417

38. Murphy AJ, Woollard KJ (2010) High-density lipoprotein: a potent inhibitor of inflammation. Clin Exp Pharmacol Physiol 37:710-718
39. Delia-Marina A, Garyfallia S, Petridou E (2006) Determinants of early leptin levels and later degenerative outcome. Clin Med Res 4:326-335

40. Chao W, D'Amore PA (2008) IGF2: epigenetic regulation and role in development and disease. Cytokine Growth Factor Rev 19:111-120

41. Hiden U, Lang I, Ghaffari-Tabrizi N et al (2009) Insulin action on the human placental endothelium in normal and diabetic pregnancy. Curr Vasc Pharmacol 7:460-466

42. Svensson AM, Ostenson CG, Bodin B, Jansson L (2005) Lack of compensatory increase in islet blood flow and islet mass in GK rats following 60\% partial pancreatectomy. J Endocrinol 184:319-327

43. Li X, Zhang L, Meshinchi S et al (2006) Islet microvasculature in islet hyperplasia and failure in a model of type 2 diabetes. Diabetes 55:2965-2973

44. Wang XH, Chen SF, Jin HM, Hu RM (2009) Differential analyses of angiogenesis and expression of growth factors in micro- and macrovascular endothelial cells of type 2 diabetic rats. Life Sci 84:240-249

45. Freathy RM, Bennett AJ, Ring SM et al (2009) Type 2 diabetes risk alleles are associated with reduced size at birth. Diabetes 58:1428-1433

46. Dinarello CA (2009) Immunological and inflammatory functions of the interleukin-1 family. Annu Rev Immunol 27:519-550

47. Duewell P, Kono H, Rayner KJ et al (2010) NLRP3 inflammasomes are required for atherogenesis and activated by cholesterol crystals. Nature 464:1357-1361

48. Chung ES, Chauhan SK, Jin Y et al (2009) Contribution of macrophages to angiogenesis induced by vascular endothelial growth factor receptor-3-specific ligands. Am J Pathol 175:1984-1992

49. Mackman N (2004) Role of tissue factor in hemostasis, thrombosis, and vascular development. Arterioscler Thromb Vasc Biol 24:1015-1022

50. Jost M, Folgueras AR, Frerart F et al (2006) Earlier onset of tumoral angiogenesis in matrix metalloproteinase-19-deficient mice. Cancer Res 66:5234-5241

51. Pirich C, Efthimiou Y, O'Grady J, Sinzinger H (1997) Hyperalphalipoproteinemia and prostaglandin I2 stability. Thromb Res $88: 41-49$

52. Zannis VI, Cole FS, Jackson CL, Kurnit DM, Karathanasis SK (1985) Distribution of apolipoprotein A-I, C-II, C-III, and E mRNA in fetal human tissues. Time-dependent induction of apolipoprotein $\mathrm{E}$ mRNA by cultures of human monocyte-macrophages. Biochemistry 24:4450-4455

53. Bonizzi G, Karin M (2004) The two NF-kappaB activation pathways and their role in innate and adaptive immunity. Trends Immunol 25:280-288

54. Bazaes RA, Salazar TE, Pittaluga E et al (2003) Glucose and lipid metabolism in small for gestational age infants at 48 hours of age. Pediatrics 111:804-809

55. Le Clair C, Abbi T, Sandhu H, Tappia PS (2009) Impact of maternal undernutrition on diabetes and cardiovascular disease risk in adult offspring. Can J Physiol Pharmacol 87:161-179 